\title{
Core-Shell Beads as Microreactors for Phylogrouping of $E$. coli Strains
}

\author{
Lena Gorgannezhad ${ }^{1,2}{ }^{\oplus}$, Kamalalayam Rajan Sreejith ${ }^{1}$, Melody Christie ${ }^{2}$, Jing Jin ${ }^{1}(\mathbb{D}$, \\ Chin Hong Ooi ${ }^{1}\left(\mathbb{D}\right.$, Mohammad Katouli ${ }^{3}{ }^{(D}$, Helen Stratton ${ }^{2}$ and Nam-Trung Nguyen ${ }^{1, *(D)}$ \\ 1 Queensland Micro- and Nanotechnology Centre, Nathan Campus, Griffith University, 170 Kessels Road, \\ Brisbane, QLD 4111, Australia; lena.gorgannezhad@griffithuni.edu.au (L.G.); \\ sreejith.kamalalayamrajan@griffithuni.edu.au (K.R.S.); jing.jin3@griffithuni.edu.au (J.J.); \\ c.ooi@griffith.edu.au (C.H.O.) \\ 2 School of Environment and Science, Nathan Campus, Griffith University, 170 Kessels Road, \\ Brisbane, QLD 4111, Australia; m.christie@griffith.edu.au (M.C.); h.stratton@griffith.edu.au (H.S.) \\ 3 Genecology Research Centre, School of Health and Sports Science, University of the Sunshine Coast, \\ Maroochydore DC, Queensland 4558, Australia; mkatouli@usc.edu.au \\ * Correspondence: nam-trung.nguyen@griffith.edu.au
}

Received: 16 June 2020; Accepted: 4 August 2020; Published: 7 August 2020

check for updates

\begin{abstract}
Multiplex polymerase chain reaction (PCR) is an effective tool for simultaneous detection of target genes. Nevertheless, their use has been restricted due to the intrinsic interference between primer pairs. Performing several single PCRs in an array format instead of a multiplex PCR is a simple way to overcome this obstacle. However, there are still major technical challenges in designing a new generation of single PCR microreactors with a small sample volume, rapid thermal cycling, and no evaporation during amplification. We report a simple and robust core-shell bead array for a series of single amplifications. Four core-shell beads with a polymer coating and PCR mixture were synthesized using liquid marble formation and subsequent photo polymerization. Each bead can detect one target gene. We constructed a customised system for thermal cycling of these core-shell beads. Phylogrouping of the E. coli strains was carried out based on the fluorescent signal of the core-shell beads. This platform can be a promising alternative for multiplex nucleic acid analyses due to its simplicity and high throughput. The platform reported here also reduces the cycling time and avoids evaporation as well as contamination of the sample during the amplification process.
\end{abstract}

Keywords: PCR; liquid marble; core-shell bead; phylogrouping; simultaneous detection

\section{Introduction}

Escherichia coli (E. coli) is a bacterium that is frequently found in the environment and the intestine of humans and some animals. While most strains of E. coli are not harmful, some strains are pathogenic and can cause infectious diseases such as pneumonia, urinary tract infections, diarrhea, gastrointestinal, and extra-intestinal infections [1,2]. Phylogenetic studies of E. coli strains identified four main groups comprising A, B1, B2, and D [3,4]. The distribution within these groups is related to the host origin of the strains. Phylogroups B2 and D demonstrated to include virulent strains, expressing several virulence factors $[5,6]$. However, phylogroups $\mathrm{A}$ and B1 are the most predominant and commensal groups in human and animal [7-9].

Over the past few decades, a broad range of conventional genotyping methods were employed for the characterisation of E. coli phylogroups. These approaches can be divided into two main groups: (i) digestion-based methods such as restriction fragment length evaluation, pulsed-field gel electrophoresis (PFGE) [10]; and (ii) PCR-based methods including enterobacterial repetitive intergenic consensus 
sequence (ERIC)- PCR [11,12], randomly amplified polymorphic DNA (RAPD) analysis only [13] or coupled with biochemical fingerprinting [14], ribotyping analysis [15], and multiplex phylogrouping PCR $[16,17]$. The major bottlenecks of these conventional approaches are the time-consuming and elaborate laboratory procedures and the corresponding equipment. PFGE is a genotyping technique that is used for digesting bacterial nucleic acids by restriction enzymes followed by fragmented DNA analysis. This digestion may result in DNA patterns even with a very low number of bands. The ease of interpretation in PFGE makes it a handy approach for genotyping of bacterial isolates. Nevertheless, this method has a long processing time of 5 to 6 days as required for isolation and digestion of DNA before electrophoresis [18]. (ERIC)-PCR is a powerful, rapid, and profitable fingerprinting method to discriminate the different strains. However, the large number of protocol steps affects its repeatability and speed. Furthermore, this method needs gel electrophoresis as a mandatory step for identification $[19,20]$. RAPD is a popular genotyping approach. This method amplifies a large template of genomic DNA using short and arbitrary primers, generating highly polymorphic fragments profiles. These fragmented DNA can be used as microbial identification fingerprints. These profiles can be quickly analysed without PFGE [21], even though various normalization methods have been developed to solve the reproducibility problem of this method [22]. PCR ribotyping has also been introduced as a useful method for bacterial phylogrouping that relies on phylogenetic analyses of existing rRNA [23].

Multiplex (tri/tetra plex) is another common PCR-based molecular technique for E. coli phylogrouping. In this method, the specific primers are simultaneously used for the amplification of 4 genes whose presence/absence produces a genotype, which identifies an isolate as a member of one of 8 different phylogroups [4,24]. Among the above-mentioned approaches, PCR ribotyping and multiplex PCR have been adopted in many laboratories as the ideal approach for E. coli typing. However, there are still some obstacles that limit their application. For example, agarose gel-based DNA separation technique is a necessary step for both assays. Conventional agarose gel-based technique has limitations such as inter-laboratory difference in explaining banding patterns, time-consuming and complex processes, and the need for skilled personnel as well as expensive equipment $[25,26]$. Although some previously reported systems such as labelled probes have been also used for direct target gene identification without the need for electrophoresis, the implementation of these probe-based sensors is time consuming and requires multiple PCR product handling steps [27].

Microfluidics was successfully utilised for nucleic acid amplification [28]. Lab-on-a-chip systems attracted a great deal of attention from the research community and the industry due to their advantages such as real-time analysis, miniaturization, portability, and high throughput [29]. Microfluidic devices also have other distinct advantages such as providing multiplex experiments, consuming a small amount of samples, and integration of optical components in the same device [30-34]. Furthermore, droplet-based microfluidics allow for the precise control of sample volume with temperature control for actuation [35], sorting [36], and thermal cycling for amplification of nucleic acids. However, the major drawbacks of droplet-based microfluidics are complex fabrication processes, difficulties in sealing and valving, evaporation, and inconsistency. Furthermore, the design and fabrication of the corresponding devices need specialised instruments and expertise [37]. Thus, there is a need for novel real-time amplification methods for phylogrouping with features such as low cost, high speed, simple operation, and high sensitivity.

Liquid marble, a liquid droplet encapsulated by hydrophobic powder, can be an alternative platform to address the above issues of conventional methods. The powder coating prevents the direct contact between the core liquid and the surrounding, thus reducing contamination [38]. Liquid marble can maintain its stability on a solid surface, making handling a liquid becomes handling a solid [39]. The porous coating allows for introducing and removing reagents and reaction products into and from a liquid marble. Due to the small dimension of a liquid marble, the reagent consumption can be reduced [40,41], particularly as for chemical and biological applications [42]. More recently, Sreejith et al. [43] used liquid marbles as reactors for DNA amplification. Polytetrafluoroethylene (PTFE) was used as the hydrophobic coating. However, the overall thermal cycling time was limited 
due to the increasing rate of evaporation through the porous coating. The limited number of thermal cycles may negatively impact the amplification efficiency. The team employed a composite liquid marble as a bioreactor to reduce evaporation [44].

The present paper reports an amplification-based detection assay using four core shell beads for phylogrouping of E. coli strains. The PCR mixture was inserted into a photopolymer droplet, forming a spherical bead. The resulting liquid droplet was first embedded in a hydrophobic/oleophobic powder. The powder and the shell liquid not only provide the sterile condition for the PCR mixture, but also remarkably reduce evaporation. Moreover, solidification of the polymer transforms the liquid marble into a core-shell bead, enabling easy handling with no evaporation lost.

\section{Materials and Methods}

\subsection{Fabrication of the Core-Shell Bead}

The synthesis and characterization of a core-shell bead was reported previously [44]. The synthesis of the bead consists of four main steps. First, a silicon-type gel monolith [45] was crushed and used as the super-amphiphobic powder bed. Next, $50 \mathrm{mg}$ of camphorquinone (a photoinitiator) and $60 \mathrm{mg}$ of 94 ethyl-4-(dimethylamino) benzoate were added to $10 \mathrm{~g}$ of trimethylolpropane trimethacrylate and mixed using a stirrer at $600 \mathrm{rpm}$ for a few minutes. The prepared mixture was used as the photo polymer liquid. Subsequently, $20 \mu \mathrm{L}$ of the photopolymer was deposited on the prepared powder bed. Next, $2 \mu \mathrm{L}$ of the PCR solution was inserted into the photopolymer droplet. The composite droplet was then slowly rolled in the powder bed to create a protective amphiphobic coating (Figure 1A, steps 1-3). Finally, photopolymerization under blue light resulted in solidification of the polymer and hermetic encapsulation of the PCR master mix solution (Figure 1B). To centralize the injected PCR mixture inside the outer photopolymer droplet, photopolymerization was performed in a cylindrical drum rotating at $140 \mathrm{rpm}$ for $5 \mathrm{~min}$. Our results show that $5 \mathrm{~min}$ blue light illumination did not cause severe effect on photobleaching of the PCR products due to the short exposure time and the protective shell. However, our previous studies $[28,44]$ indicated that constant exposure on the sample during thermal cycling over a long period of time can cause photobleaching of amplicons. This issue was solved by non-continuous illumination on the beads during thermal cycling. Lastly, the coating powder was removed using mineral oil, resulting in a transparent core-shell bead. The core shell bead is a solid bead, and no shape change was observed during the thermal cycling process.

\subsection{Thermal Cycler}

A custom-built thermal cycler was developed to provide the required conditions for PCR with the core shell beads [44]. A $2 \mathrm{~cm} \times 2 \mathrm{~cm} \times 1.5 \mathrm{~cm}$ aluminium block with an embedded cartridge heating element $(0.5 \mathrm{~cm}$ diameter and $1.5 \mathrm{~cm}$ length, core-electronics) served as the heater. The aluminium block was attached to a Peltier thermoelectric cooler $(4 \mathrm{~cm} \times 4 \mathrm{~cm} \times 0.35 \mathrm{~cm}$, AUS-Electronics, NSW, Australia). An aluminium heat spreader and a cooling fan $(12 \mathrm{~V}, 3,300 \mathrm{rpm}, 7 \mathrm{~cm} \times 7 \mathrm{~cm} \times 2.5 \mathrm{~cm})$ conduct the heat out of the setup, Figure 1C. For programable thermal cycling, a PID (proportional integral derivative) controller was implemented in an Arduino UNO board to provide the closed-loop control of the temperature. This thermal cycler generated the following temperature cycles: initial denaturation at $94^{\circ} \mathrm{C}$ for $4 \mathrm{~min}, 30$ cycles of denaturation at $94^{\circ} \mathrm{C}$ for 5 sec each and annealing at $59^{\circ} \mathrm{C}$ for $20 \mathrm{sec}$ each, $5 \mathrm{~min}$ incubation at $72{ }^{\circ} \mathrm{C}$, and infinite hold at $12{ }^{\circ} \mathrm{C}$. More detail on the temperature profile during the PCR process and the control system were reported in our pervious study [44]. 
A.

1)

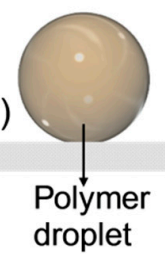

2)

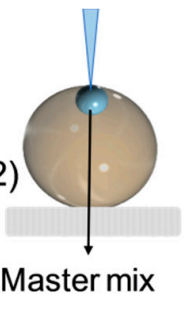

3)

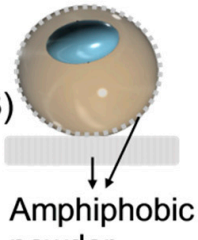
powder

D.

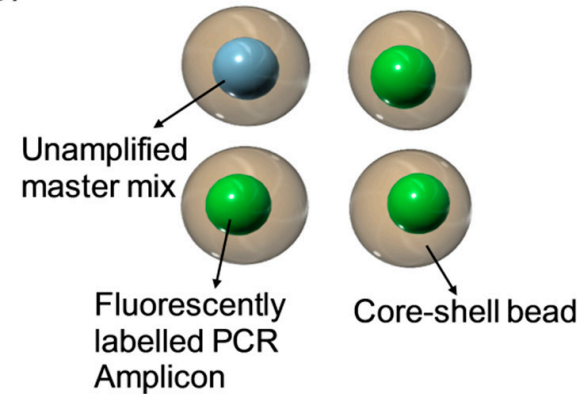

B.

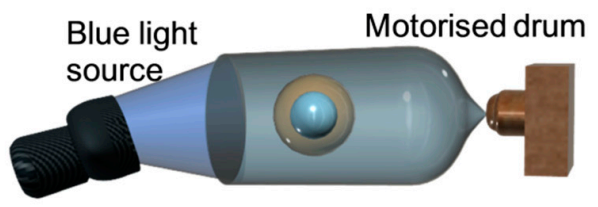

C.

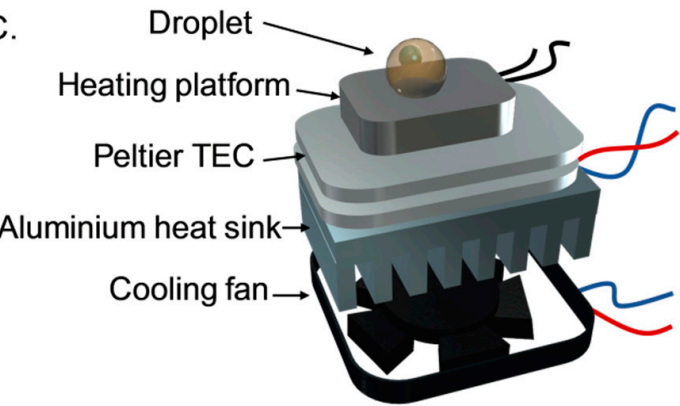

Figure 1. Operation of core-shell-bead-based polymerase chain reaction (PCR). (A) (1) Placing a droplet of photopolymer on top of an amphiphobic powder bed. (2) Inserting the PCR solution into the deposited droplet. (3) Covering the droplet with amphiphobic powder. (B) Photo polymerization of the droplet under blue light in a drum rotating at $140 \mathrm{rpm}$. (C) Transferring the generated core-shell bead to a custom-built thermal cycler for amplification. (D) Discriminating the core shell beads containing amplicons based on fluorescent intensity.

\subsection{DNA Amplification}

E. coli strains were isolated on nutrient agar plates from faeces samples in a clinical pathology lab. Single colonies were picked and grown overnight at $37^{\circ} \mathrm{C}$ in nutrient broth. Isolates were stored at $-80{ }^{\circ} \mathrm{C}$ until use in nutrient broth with $30 \%$ glycerol. The isolates were then used for phylogrouping experiment using single PCR and quadruplex PCR assays according to the method reported by Clermont et al. [46]. All samples were kept in ice before the experiment. After the sample was inserted into the bead, the sample stayed under room temperature of $23^{\circ} \mathrm{C}$ for $5 \mathrm{Min}$ before being transferred to the thermocycler. The experiments were carried out with one positive control (EC RBH2), three unknown samples, and one negative control (water). Table 1 lists the sequences of primers (forward and reverse) for the desired target genes. Single PCR utilised $20 \mu \mathrm{L}$ of the PCR mixture containing SYBR Green, template DNA, one set of primer pairs, and water. The final concentration of the SYBR Green was $1 \times$. The amounts of primer used were 20 pmol, except for AceK.f (40 pmol), ArpA1.r (40 pmol). Template DNA concentration was approximately $100 \mathrm{ng} / \mu \mathrm{L}$. A volume of $2 \mu \mathrm{L}$ of the prepared solution was inserted into the polymer droplet. After photopolymerization, the core-shell bead was placed on the customized thermal cycler. The samples underwent the aforementioned thermal cycling condition. For quadruplex PCR, the prepared PCR solution including all types of primer pairs, template DNA, and water was transferred to a conventional PCR instrument (Biorad CFX Connect, NSW, Australia) for thermal cycling. The final concentrations of the reagents were mentioned previously. The resulting amplicons were subsequently run on $2 \%$ agarose gel electrophoresis and followed by exposure of the UV transilluminator (Bio-Rad). E. coli strains were isolated by a clinical pathology lab in Turkey as part of the routine analyses of clinical samples. No clinical samples were directly collected from any patients, nor were the isolated strains part of any projects; therefore, there is no need for ethics approval for further testing them. 
Table 1. Primer sequences and sizes of amplicons employed in the phylo-grouping experiment.

\begin{tabular}{cccc}
\hline Target Gene & Primer ID & Sequence (5-3') & PCR Product Size (bp) \\
\hline \multirow{2}{*}{ chuA } & chuA.1b & ATGGTACCGGACGAACCAAC & \multirow{2}{*}{288} \\
& chuA.2 & TGCCGCCAGTACCAAAGACA & \multirow{2}{*}{211} \\
yjaA & yjaA.1b & CAAACGTGAAGTGTCAGGAG & \multirow{2}{*}{152} \\
& yjaA.2b & AATGCGTTCCTCAACCTGTG & \multirow{2}{*}{ TspE4C2 } \\
TspE4C2.1b & CACTATTCGTAAGGTCATCC & \\
TspE4C2.2b & AGTTTATCGCTGCGGGTCGC & \\
\hline
\end{tabular}

\subsection{Optical Detection}

For real-time analysis of the amplification, we developed a fluorescent detection platform as depicted in Figure 2. The range of the fluorescent excitation wavelengths was 450-490 nm (Blue light). The range of emission wavelengths was 520-560 nm (Green). A blue LED light (450-490nm) (1500mCd, Jaycar, Brisbane, Australia) served as the excitation source. Fluorescence measurement was performed at the end of the thermal cycles, and the fluorescent signal was recorded by a CMOS camera (EO-5012C, Edmund Optic, Singapore) equipped with a $0.5 \times$ telecentric lens (Edmund industrial Optics-63074). A green optical filter (520-560 nm) was also used for the emitted light to enhance the signal to noise ratio. The intensity of the emitted light provides information about the amplification potency.

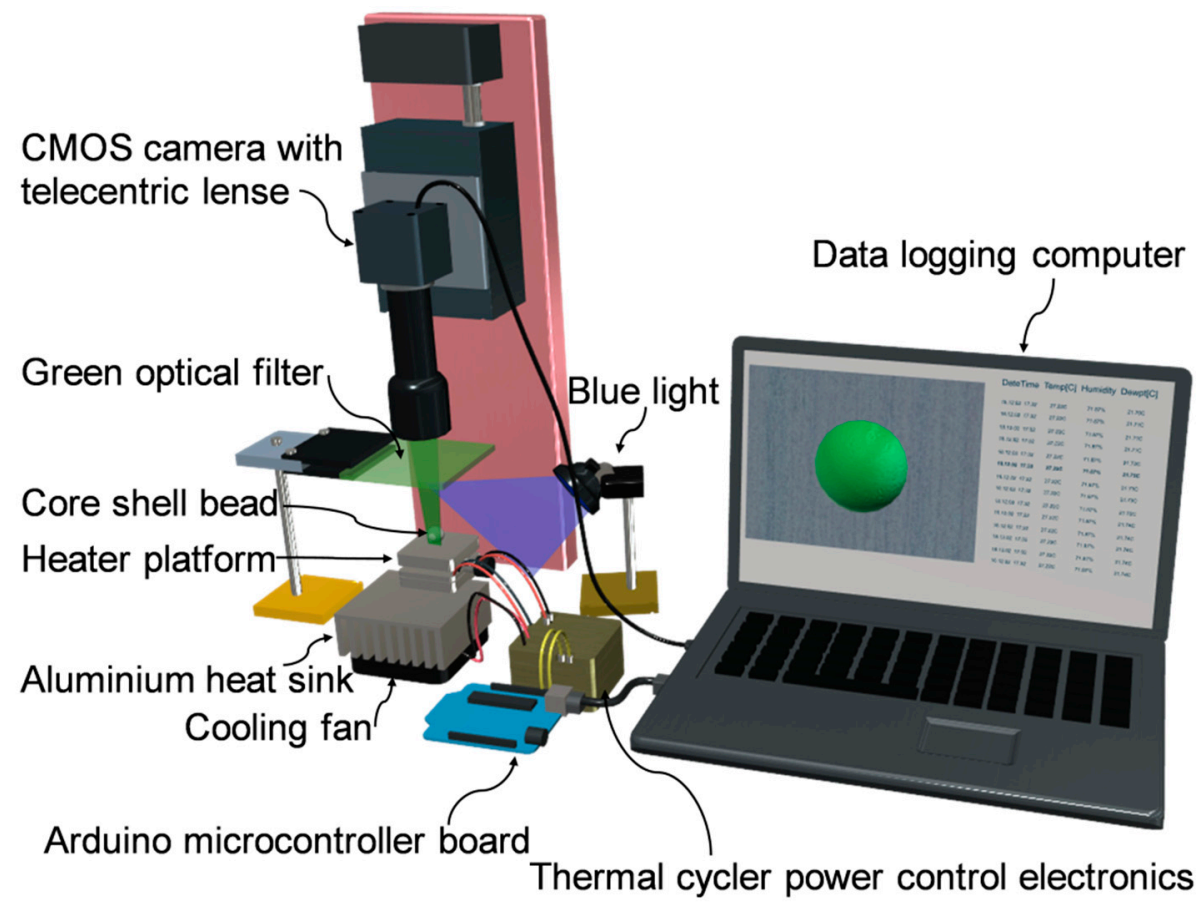

Figure 2. Schematic of the experimental setup.

\section{Results and Discussion}

\subsection{Assay Principle}

Using a core-shell bead as a microreactor (Figure 1), we demonstrate here an amplification-based assay for tracing marker genes and phylogrouping E. coli strains. For the phylogrouping experiment, four core-shell beads were fabricated. Each bead contained $2 \mu \mathrm{L}$ PCR solution with a particular set of primer to detect one marker gene. The resulting core-shell beads were subsequently located on a customised PCR device for thermal cycling. A separator made of polydimethylsiloxane (PDMS) was 
used to prevent the beads from sticking to the heater during the thermal cycling process. The experiment was performed in a dark laboratory environment. The blue LED light source was employed to match the excitation wavelength. The light was manually turned on at five-cycle intervals. Experiments were repeated four times, one for positive control and three for unknown samples. Compared to the traditional phylogrouping method (PCR and subsequent gel electrophoresis), which take almost $3 \mathrm{~h}$, our assay was carried out in around $1.5 \mathrm{~h}$. One experiment with an unknown sample was repeated three times to test the reproducibility of the assay. Satisfactory results were achieved as shown in Figure S2. Moreover, based on our sensitivity measurements shown in Figure S3, the limit of detection (LOD) for the assay was $100 \mathrm{ng} / \mu \mathrm{L}$.

\subsection{Phylogrouping Using Core Shell Beads}

We used core-shell beads of A, B, C, and D for the analysis of chuA, arpA, TspE4C2, and yjaA genes, respectively, to demonstrate the capability of our method for analysing clinical (control/unknown) samples. The numerical values of the fluorescent intensities of the amplicons inside the core shell beads were evaluated using ImageJ. The values were normalised as

$$
I_{\mathrm{sc}}^{*}=\left(I_{\mathrm{sc}}-I_{\mathrm{s} 0}\right) / I_{\max }
$$

where $I_{\mathrm{sc}}$ is the fluorescence intensity of the sample measured in a given cycle, $I_{\mathrm{s} 0}$ is the fluorescence intensity of that sample at the beginning of the thermal cycling process, and $I_{\max }$ is the maximum fluorescent intensity measured between all samples. Figure S1 shows the representative images of the beads during an experiment to illustrate the increase in fluorescence intensity over the thermal cycling process. Figure 3 shows the amplification curves of the core-shell beads. Increasing the cycle number enhances the normalised fluorescence intensity of bead comprising target gene and related primer set, indicating a positive polymerase chain reaction within the bead. The lack of target gene inside a bead results in no significant fluorescence over thermal cycling. Each strain can be genotyped based on the presence/absence of the four genes in four beads. Figure 3 (I) shows phylogrouping of a positive control (EC RBH2). Beads A, C, and D show positive amplification. However, bead B has no significant amplification. The obtained genotype $(+-++)$ indicates chuA,$+ \operatorname{arpA}-$, TspE4C2 + , and yjaA + , and subsequently an isolate is attributed to a phylo-group of $\mathrm{B} 2$. In addition to one positive control, three unknown samples were also evaluated using this assay. The corresponding phylo-groups are depicted in Table 2. Two of the E. coli isolates could not be exactly assigned to one of the known phylo-groups and reported as unknown strains. The multi-locus sequence type (MLST) technique [46] is required to assign these strain fractions.

Table 2. Genotypes of isolated E. coli samples.

\begin{tabular}{cccccc}
\hline Experiment ID & Bead A (chuA) & Bead B (arpA) & $\begin{array}{c}\text { Bead C } \\
(\text { TspE4C2) }\end{array}$ & Bead D (yjaA) & Phylo-Group \\
\hline I & + & - & + & + & B2 \\
II & - & - & + & + & Unknown \\
III & - & - & + & - & Clade I/II \\
IV & - & & & Unknown \\
\hline
\end{tabular}


I)

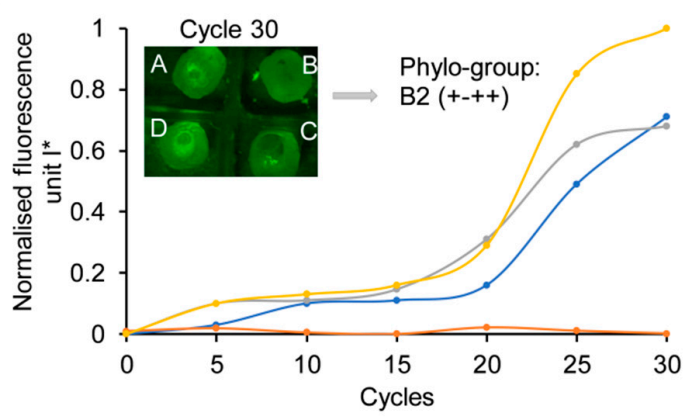

IV)

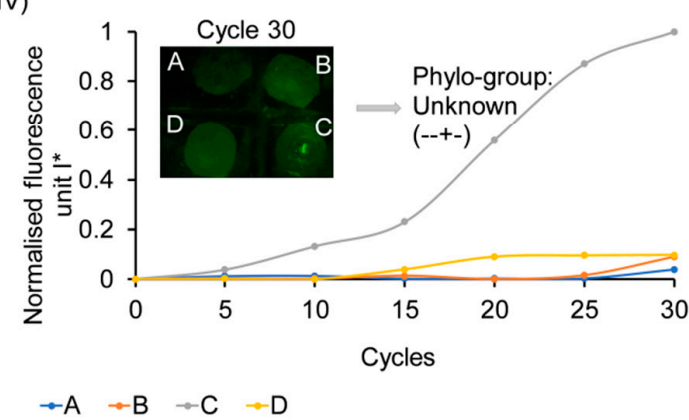

II)

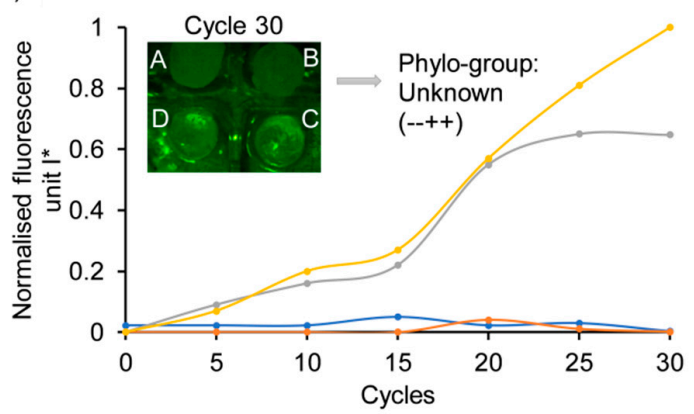

III)

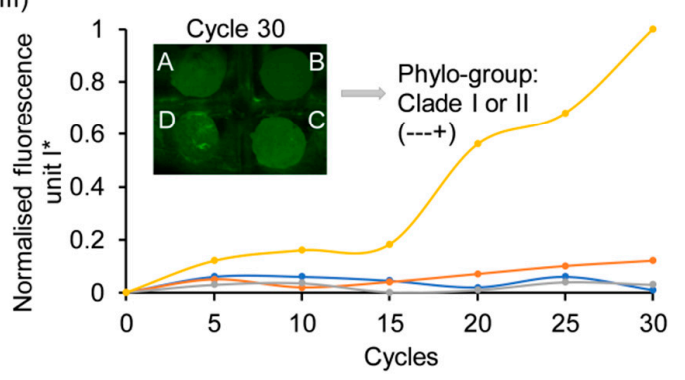

Figure 3. Amplification plot and phylogrouping of E. coli strains using core shell beads. (I) Positive control; (II-IV) unknown samples; A, B, C, and D beads containing specific set of primers to detect chuA, arpA, TspE4C2, yjaA genes individually; and images of core shell beads, are provided as insets.

\subsection{Validation of Phylogrouping by Gel Electrophoresis}

We used quadruplex PCR and gel electrophoresis to verify the phylogrouping results of the samples in a commercial platform. Figure 4 shows the result of Sybr-Green quadruplex PCR of positive control (EC RBH2), negative control (water), and unknown samples. Evaluation of the banding pattern on the gel showed three separate bands of $288 \mathrm{bp}, 211 \mathrm{bp}$, and $152 \mathrm{bp}$ for chuA, yjaA, and TspE4C2 genes, respectively. Lane 2 assigns to $\mathrm{B} 2$ strain of E. coli that possess chuA, yjaA, and TspE4C2 genes. E.clade I/II strain just shows amplification of yjaA gene (lane 5). The remaining genotypes (lane 4 and 6) cannot be classified into any distinct phylo-group. These results validate the accuracy of the amplification using core-shell beads.

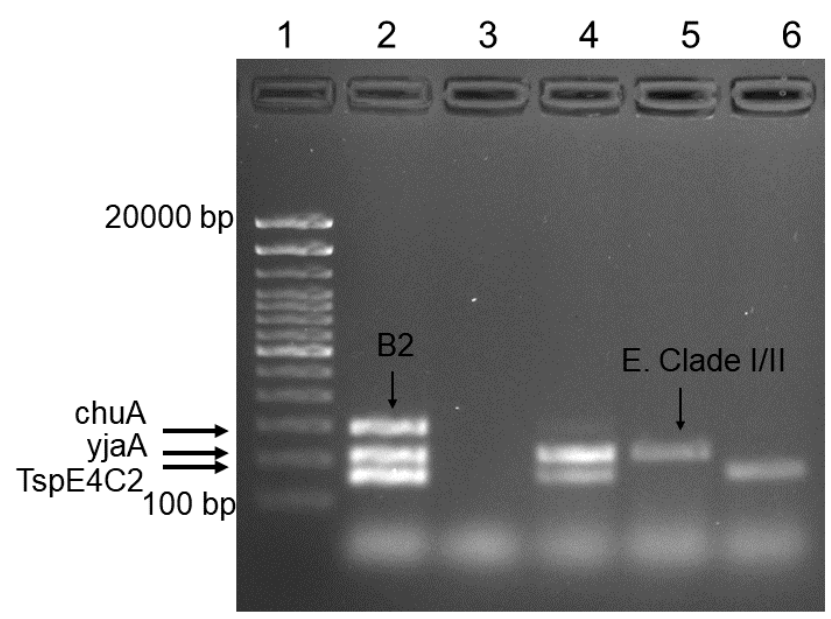

Figure 4. Agarose gel electrophoresis of amplicons from the Sybr-Green quadruplex PCR for confirmation. Lane 1: DNA ladder (100-20,000 bp). Lane 2: positive control (EC RBH2). Lane 3: negative control (water). Lane 4 to 6: unknown samples. 


\section{Conclusions}

Four core-shell polymer beads containing the PCR mixture were prepared by forming a liquid marble and subsequent photo polymerisation. A customised thermal cycler was developed to provide temperature control of the core-shell beads. Thermal cycling of the core-shell beads as PCR microreactor was performed for phylogrouping of the E. coli strains. This method potentially resolves the issues of conventional multiplex PCR in a single tube such as the intrinsic interference and the competition among primers, the requirement for optimized multiplex PCR protocols, and the difficulty in simultaneously detecting a large number of targets. Our assay demonstrated the following advantages: (i) simple fabrication and operation; (ii) fully sealed PCR microreactor that eliminates volume loss due to evaporation and minimises the risk of contamination; and (iii) reducing the PCR mixture volume to $2 \mu \mathrm{L}$, which decreases the cycle time. In summary, the core-shell bead platform reported here is capable of full functionality of a PCR assay due to its simplicity and potentially high throughput. This PCR microreactor can be employed in nucleic acid studies for clinical or environmental diagnostics. We believe that our method has a huge potential for commercialisation due to the reduction of expensive lab-based equipment and labour costs. Further development will focus on the automation of the platform, making it more user-friendly. Current manual fabrication of beads for a large number of samples is labour intensive. Thus, automation of bead preparation, partitioning of the PCR solution, and delivery into the fabricated beads can increase the speed and efficiency of the assay. Moreover, this platform has the potential to be fully integrated with other steps such as sample preparation, DNA extraction, amplification, and optical detection for the identification of bacteria as well as other basic research and translational applications.

Supplementary Materials: The following are available online at http://www.mdpi.com/2072-666X/11/8/761/s1, Figure S1: Photographs of one set of the beads during thermal cycling, Figure S2: Reproducibility test of amplification for an unknown E. coli strain using core shell beads, Figure S3: Sensitivity test of amplification for one unknown E. coli strain with different concentrations using core shell beads.

Author Contributions: Conceptualization, N.-T.N., L.G., and K.R.S.; methodology, L.G., K.R.S., M.C., J.J., and C.H.O.; validation, L.G.; formal analysis, L.G.; investigation, L.G.; resources, N.-T.N.; data curation, L.G.; writing-original draft preparation, L.G.; writing-review and editing, N.-T.N., L.G., K.R.S., M.K., and H.S.; visualization, L.G.; supervision, N.-T.N., H.S.; project administration, N.-T.N., L.G. All authors have read and agreed to the published version of the manuscript.

Funding: This research was funded by Australian Research Council through the grant DP170100277. Parts of the work were carried out at the Queensland node, Griffith University, of the Australian National Fabrication Facility, a company established under the National collaborative Research Infrastructure Strategy to provide nano and microfabrication facilities for Australia's researchers.

Acknowledgments: This work was supported by the higher degree research scholarship (GUIPRS and IPRS) to L.G. from the Griffith University. N.-T.N.

Conflicts of Interest: The authors declare no conflict of interest.

\section{References}

1. Chen, S.L.; Wu, M.; Henderson, J.P.; Hooton, T.M.; Hibbing, M.E.; Hultgren, S.J.; Gordon, J.I. Genomic diversity and fitness of E. coli strains recovered from the intestinal and urinary tracts of women with recurrent urinary tract infection. Sci. Transl. Med. 2013, 5, 184ra160. [CrossRef] [PubMed]

2. Phillips-Houlbracq, M.; Ricard, J.-D.; Foucrier, A.; Yoder-Himes, D.; Gaudry, S.; Bex, J.; Messika, J.; Margetis, D.; Chatel, J.; Dobrindt, U. Pathophysiology of Escherichia coli pneumonia: Respective contribution of pathogenicity islands to virulence. Int. J. Med. Microbiol. 2018, 308, 290-296. [CrossRef] [PubMed]

3. Herzer, P.J.; Inouye, S.; Inouye, M.; Whittam, T.S. Phylogenetic distribution of branched RNA-linked multicopy single-stranded DNA among natural isolates of Escherichia coli. J. Bacteriol. 1990, 172, 6175-6181. [CrossRef] [PubMed]

4. Clermont, O.; Bonacorsi, S.; Bingen, E. Rapid and simple determination of the Escherichia coli phylogenetic group. Appl. Environ. Microbiol. 2000, 66, 4555-4558. [CrossRef] 
5. Bingen, E.; Picard, B.; Brahimi, N.; Mathy, S.; Desjardins, P.; Elion, J.; Denamur, E. Phylogenetic analysis of Escherichia coli strains causing neonatal meningitis suggests horizontal gene transfer from a predominant pool of highly virulent B2 group strains. J. Infect. Dis. 1998, 177, 642-650. [CrossRef]

6. Picard, B.; Garcia, J.S.; Gouriou, S.; Duriez, P.; Brahimi, N.; Bingen, E.; Elion, J.; Denamur, E. The link between phylogeny and virulence in Escherichia coli extraintestinal infection. Infect. Immun. 1999, 67, 546-553. [CrossRef]

7. Duriez, P.; Clermont, O.; Bonacorsi, S.; Bingen, E.; Chaventré, A.; Elion, J.; Picard, B.; Denamur, E. Commensal Escherichia coli isolates are phylogenetically distributed among geographically distinct human populations. Microbiology 2001, 147, 1671-1676. [CrossRef]

8. Gordon, D.M.; Cowling, A. The distribution and genetic structure of Escherichia coli in Australian vertebrates: Host and geographic effects. Microbiology 2003, 149, 3575-3586. [CrossRef]

9. Escobar-Páramo, P.; Le Menac'H, A.; Le Gall, T.; Amorin, C.; Gouriou, S.; Picard, B.; Skurnik, D.; Denamur, E. Identification of forces shaping the commensal Escherichia coli genetic structure by comparing animal and human isolates. Environ. Microbiol. 2006, 8, 1975-1984. [CrossRef]

10. Lautenbach, E.; Bilker, W.B.; Tolomeo, P.; Maslow, J.N. Impact of diversity of colonizing strains on strategies for sampling Escherichia coli from fecal specimens. J. Clin. Microbiol. 2008, 46, 3094-3096. [CrossRef]

11. Moreno, E.; Andreu, A.; Pigrau, C.; Kuskowski, M.A.; Johnson, J.R.; Prats, G. Relationship between Escherichia coli strains causing acute cystitis in women and the fecal E. coli population of the host. J. Clin. Microbiol. 2008, 46, 2529-2534. [CrossRef] [PubMed]

12. Moreno, E.; Johnson, J.R.; Pérez, T.; Prats, G.; Kuskowski, M.A.; Andreu, A. Structure and urovirulence characteristics of the fecal Escherichia coli population among healthy women. Microbe. Infect. 2009, 11, 274-280. [CrossRef] [PubMed]

13. Nowrouzian, F.L.; Wold, A.E.; Adlerberth, I. Escherichia coli strains belonging to phylogenetic group B2 have superior capacity to persist in the intestinal microflora of infants. J. Infect. Dis. 2005, 191, 1078-1083. [CrossRef]

14. Vollmerhausen, T.L.; Ramos, N.L.; Gündoğdu, A.; Robinson, W.; Brauner, A.; Katouli, M. Population structure and uropathogenic virulence-associated genes of faecal Escherichia coli from healthy young and elderly adults. J. Med. Microbiol. 2011, 60, 574-581. [CrossRef] [PubMed]

15. Anderson, M.A.; Whitlock, J.E.; Harwood, V.J. Diversity and distribution of Escherichia coli genotypes and antibiotic resistance phenotypes in feces of humans, cattle, and horses. Appl. Environ. Microbiol. 2006, 72, 6914-6922. [CrossRef] [PubMed]

16. Escobar-Páramo, P.; Grenet, K.; Le Menac'h, A.; Rode, L.; Salgado, E.; Amorin, C.; Gouriou, S.; Picard, B.; Rahimy, M.C.; Andremont, A. Large-scale population structure of human commensal Escherichia coli isolates. Appl. Environ. Microbiol. 2004, 70, 5698-5700. [CrossRef] [PubMed]

17. Staji, H. Detection of enterohemorrhagic Escherichia coli related genes in E. coli strains belonging to B2 phylogroup isolated from urinary tract infections in combination with antimicrobial resistance phenotypes. JMB 2017, 6, 36-44.

18. Sperner, B.; Schalch, B.; Eisgruber, H.; Stolle, A. Short protocol for pulsed field gel electrophoresis of a variety of Clostridia species. FEMS Immunol. Med. Microbiol. 1999, 24, 287-292. [CrossRef]

19. Mouwen, D.; Weijtens, M.; Capita, R.; Alonso-Calleja, C.; Prieto, M. Discrimination of enterobacterial repetitive intergenic consensus PCR types of Campylobacter coli and Campylobacter jejuni by Fourier transform infrared spectroscopy. Appl. Environ. Microbiol. 2005, 71, 4318-4324. [CrossRef]

20. Ranjbar, R.; Tabatabaee, A.; Behzadi, P.; Kheiri, R. Enterobacterial repetitive intergenic consensus polymerase chain reaction (ERIC-PCR) genotyping of Escherichia coli strains isolated from different animal stool specimens. Iran. J. Pathol. 2017, 12, 25. [CrossRef]

21. Kumar, N.S.; Gurusubramanian, G. Random amplified polymorphic DNA (RAPD) markers and its applications. Sci. Vis. 2011, 11, 116-124.

22. Lin, W.-J.; Tung, C.-Y.; Yen, M.-Y.; Chan, Y.-J.; Lin, C.-H.; Hsueh, P.-R. A novel target pathogen identification and tracking system using capillary electrophoresis-random amplified polymorphic DNA. Sci. Rep. 2018, 8, 15365. [CrossRef] [PubMed] 
23. Huber, C.A.; Foster, N.F.; Riley, T.V.; Paterson, D.L. Challenges for standardization of Clostridium difficile typing methods. J. Clin. Microbiol. 2013, 51, 2810-2814. [CrossRef] [PubMed]

24. Roohollah, K.; Naser, H.; Mehrouz, D. Efficacy evaluation of four different culture and PCR-based methods of Escherichia coli detection in water samples. Adv. Environ. Biol. 2013, 7, 2689-2694.

25. Diego, J.G.-B.; Fernández-Soto, P.; Crego-Vicente, B.; Alonso-Castrillejo, S.; Febrer-Sendra, B.; Gómez-Sán chez, A.; Vicente, B.; López-Abán, J.; Muro, A. Progress in loop-mediated isothermal amplification assay for detection of Schistosoma mansoni DNA: Towards a ready-to-use test. Sci. Rep. 2019, 9, 1-11. [CrossRef]

26. Fawley, W.N.; Knetsch, C.; MacCannell, D.R.; Harmanus, C.; Du, T.; Mulvey, M.R.; Paulick, A.; Anderson, L.; Kuijper, E.; Wilcox, M.H. Development and validation of an internationally-standardized, high-resolution capillary gel-based electrophoresis PCR-ribotyping protocol for Clostridium difficile. PLoS ONE 2015, 10, e0118150. [CrossRef]

27. Mackay, I.M.; Arden, K.E.; Nitsche, A. Real-time PCR in virology. Nucleic Acids Res. 2002, 30, $1292-1305$. [CrossRef]

28. Gorgannezhad, L.; Sreejith, K.R.; Zhang, J.; Kijanka, G.; Christie, M.; Stratton, H.; Nguyen, N.-T. Microfluidic array chip for parallel detection of waterborne bacteria. Micromachines 2019, 10, 883. [CrossRef]

29. Nguyen, N.-T.; Hejazian, M.; Ooi, C.H.; Kashaninejad, N. Recent advances and future perspectives on microfluidic liquid handling. Micromachines 2017, 8, 186. [CrossRef]

30. Gorgannezhad, L.; Stratton, H.; Nguyen, N.-T. Microfluidic-based nucleic acid amplification systems in microbiology. Micromachines 2019, 10, 408. [CrossRef]

31. Gorgannezhad, L.; Umer, M.; Islam, M.N.; Nguyen, N.-T.; Shiddiky, M.J. Circulating tumor DNA and liquid biopsy: Opportunities, challenges, and recent advances in detection technologies. Lab Chip 2018, 18, 1174-1196. [CrossRef] [PubMed]

32. Huang, G.; Huang, Q.; Xie, L.; Xiang, G.; Wang, L.; Xu, H.; Ma, L.; Luo, X.; Xin, J.; Zhou, X. A rapid, low-cost, and microfluidic chip-based system for parallel identification of multiple pathogens related to clinical pneumonia. Sci. Rep. 2017, 7, 6441. [CrossRef] [PubMed]

33. Gorgannezhad, L.; Umer, M.; Kamal Masud, M.; Hossain, M.S.A.; Tanaka, S.; Yamauchi, Y.; Salomon, C.; Kline, R.; Nguyen, N.T.; Shiddiky, M.J. Detection of FGFR2: FAM76A fusion gene in circulating tumor RNA based on catalytic signal amplification of graphene oxide-loaded magnetic nanoparticles. Electroanalysis 2018, 30, 2293-2301. [CrossRef]

34. Song, C.; Nguyen, N.-T.; Tan, S.-H.; Asundi, A.K. Modelling and optimization of micro optofluidic lenses. Lab Chip 2009, 9, 1178-1184. [CrossRef] [PubMed]

35. Jiao, Z.; Huang, X.; Nguyen, N.-T.; Abgrall, P. Thermocapillary actuation of droplet in a planar microchannel. Microfluid. Nanofluidics 2008, 5, 205-214. [CrossRef]

36. Yap, Y.-F.; Tan, S.-H.; Nguyen, N.-T.; Murshed, S.S.; Wong, T.-N.; Yobas, L. Thermally mediated control of liquid microdroplets at a bifurcation. J. Phys. D Appl. Phys. 2009, 42, 065503. [CrossRef]

37. Duncombe, T.A.; Tentori, A.M.; Herr, A.E. Microfluidics: Reframing biological enquiry. Nat. Rev. Mol. Cell Biol. 2015, 16, 554-567. [CrossRef]

38. Bormashenko, E.; Balter, R.; Aurbach, D. Micropump based on liquid marbles. Appl. Phys. Lett. 2010, 97, 091908. [CrossRef]

39. Mahadevan, L.; Pomeau, Y. Rolling droplets. Phys. Fluids. 1999, 11, 2449-2453. [CrossRef]

40. Tian, J.; Arbatan, T.; Li, X.; Shen, W. Liquid marble for gas sensing. Chem. Commun. 2010, 46, 4734-4736. [CrossRef]

41. Xue, Y.; Wang, H.; Zhao, Y.; Dai, L.; Feng, L.; Wang, X.; Lin, T. Magnetic liquid marbles: A "precise" miniature reactor. Adv. Mater. 2010, 22, 4814-4818. [CrossRef] [PubMed]

42. Leite, Á.J.; Oliveira, N.M.; Song, W.; Mano, J.F. Bioactive hydrogel marbles. Sci. Rep. 2018, 8, 15215. [CrossRef] [PubMed]

43. Sreejith, K.R.; Gorgannezhad, L.; Jin, J.; Ooi, C.H.; Stratton, H.; Dao, D.V.; Nguyen, N.-T. Liquid marbles as biochemical reactors for the polymerase chain reaction. Lab Chip 2019, 19, 3220-3227. [CrossRef] [PubMed]

44. Sreejith, K.R.; Gorgannezhad, L.; Jin, J.; Ooi, C.H.; Takei, T.; Hayase, G.; Stratton, H.; Lamb, K.; Shiddiky, M.; Dao, D.V.; et al. Core-shell beads made by composite liquid marble technology as a versatile microreactor for polymerase chain reaction. Micromachines 2020, 11, 242. [CrossRef] [PubMed] 
45. Hayase, G.; Kanamori, K.; Hasegawa, G.; Maeno, A.; Kaji, H.; Nakanishi, K. A superamphiphobic macroporous silicone monolith with marshmallow-like flexibility. Angew. Chem. Int. Ed. 2013, 52, 10788-10791. [CrossRef] [PubMed]

46. Clermont, O.; Christenson, J.K.; Denamur, E.; Gordon, D.M. The C lermont Escherichia coli phylo-typing method revisited: Improvement of specificity and detection of new phylo-groups. Environ. Microbiol. Rep. 2013, 5, 58-65. [CrossRef] 